\title{
NGS as a tool for investigating spread of zoonotic bacteria: dealing with source and outhreak genetic variation in a spatio-temporal context
}

\author{
Robert Söderlund ${ }^{1,2} \llbracket$, Tomas Jinnerot ${ }^{2}$, Adrien Janssens ${ }^{1}$, Erik Bongcam-Rudloff ${ }^{1}$ \\ 'Swedish University of Agricultural Sciences, Uppsala, Sweden \\ ${ }^{2}$ National Veterinary Institute, Sweden

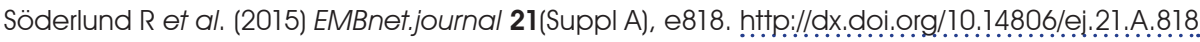

Next generation sequencing (NGS) is increasingly the method of choice for tracing transmission of zoonotic bacterial disease agents; infections that are spread to humans from animals. Establishing the link to a source of infection is dependent on an implicit or explicit definition of a source population from which the outbreak strain originates. The link is also based on assumptions about the relevance of a sampling occasion for the time the infection event took place.

While these problems are the same regardless of the method used, they are magnified by the high resolution of NGS-based typing. In particular, exact genotypic matches are rare leading to a much greater challenge in comparing genetic distances, drawing conclusions from them and communicating the quantitative results to a non-specialist audience.
We are currently conducting a series of studies to better define the source of genetic variation found in bacterial populations in animal reservoirs and animal feed, as well as the variation found between bacterial isolates collected from a single sampling occasion. We are also studying the spatial distribution of endemic clones in regions with dense livestock populations, and the clonal dynamics in such regions. Our focus is on enterohaemorrhagic E. coli (EHEC) and selected serotypes of Salmonella. The results are providing us with insights that will improve future outbreak investigations by guiding the sampling and analysis strategy as well as the interpretation of data.

\section{Acknowledgements}

This work was financed by the Swedish Board of Agriculture and the Elsa and Ivar Sandberg Foundation. 